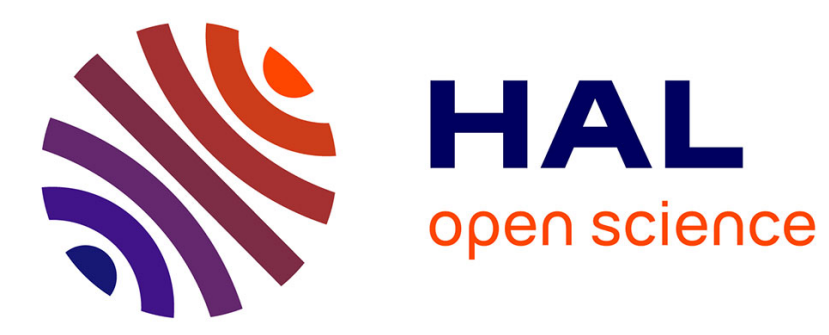

\title{
Les géographes et les frontières austro-slovènes des Alpes orientales en 1919-1920 : entre la Mitteleuropa et les Balkans.
}

\author{
Emmanuelle Boulineau
}

\section{- To cite this version:}

Emmanuelle Boulineau. Les géographes et les frontières austro-slovènes des Alpes orientales en 19191920 : entre la Mitteleuropa et les Balkans.. Revue de Géographie Alpine / Journal of Alpine Research, 2001, 4, pp.173-184. halshs-00323208

\section{HAL Id: halshs-00323208 \\ https://shs.hal.science/halshs-00323208}

Submitted on 19 Sep 2008

HAL is a multi-disciplinary open access archive for the deposit and dissemination of scientific research documents, whether they are published or not. The documents may come from teaching and research institutions in France or abroad, or from public or private research centers.
L'archive ouverte pluridisciplinaire $\mathbf{H A L}$, est destinée au dépôt et à la diffusion de documents scientifiques de niveau recherche, publiés ou non, émanant des établissements d'enseignement et de recherche français ou étrangers, des laboratoires publics ou privés. 

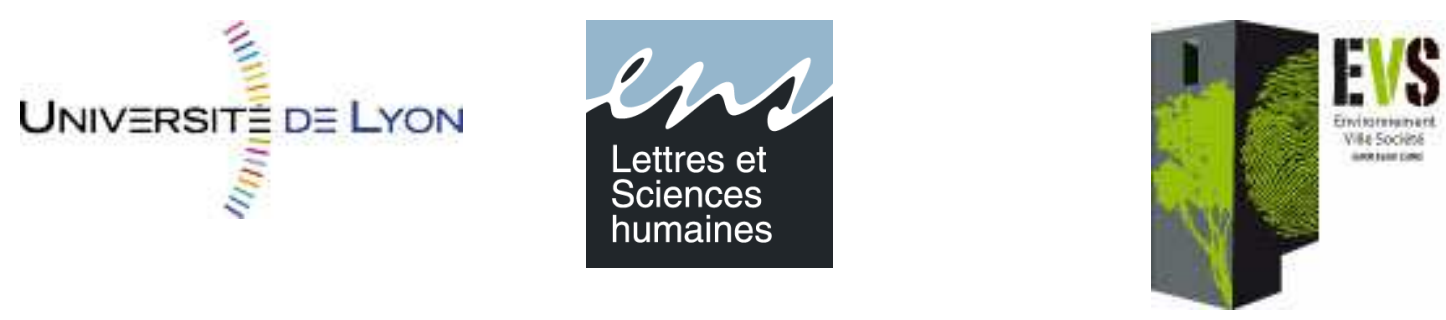

"Les géographes et les frontières austro-slovènes des Alpes orientales en 19191920 : entre la Mitteleuropa et les Balkans »,

Revue de Géographie alpine, 2001, n\%4, pp.173-184

Coordonnées de l'auteur :

Emmanuelle BOULINEAU

Maître de Conférence en Géographie

Université de Lyon, UMR 5600 Environnement ville société, ENS LSH, équipe Géophile.

15 parvis René-DescartesBP 7000

69342 Lyon cedex 07

France

Emmanuelle.boulineau@ens-lsh.fr 


\section{Les géographes et les frontières austro-slovènes des Alpes orientales en 1919- 1920 : entre la Mitteleuropa et les Balkans.}

Emmanuelle BOULINEAU, in Revue de géographie alpine, 2001- 4, pp.173-184

La fin des batailles de la première Guerre mondiale ouvre l'ère des règlements territoriaux et des questions frontalières discutés à la Conférence de Paris de 1919. Le jeune royaume des Serbes, Croates et Slovènes cherche à s'agrandir sur les dépouilles de l'empire austro-hongrois défunt à partir du cœur serbe. Tracer la frontière septentrionale du pays le long des axes de la Drave et de la Mur constitue alors un enjeu frontalier inédit. La vallée de la Drave, de Klagenfurt à Maribor (Marbourg), traverse sur $150 \mathrm{~km}$ les derniers massifs des Alpes orientales entre les Tauern au nord et la chaîne des Karawanken au sud (carte 1).À partir de Maribor, la rivière sort des "crêtes blanches et dentelées des Alpes" pour déboucher sur les "Mésopotamies pannoniques" (Ancel, 1930) et divaguer dans la plaine du Danube. On s'intéressera donc ici plus particulièrement au tracé de la frontière austro-slovène dans les derniers contreforts alpins de la Carinthie, dans ce petit bassin de Klagenfurt long de $75 \mathrm{~km}$ et large de 30 qui a cristallisé les énergies des traceurs de frontières avant de voir son sort soumis à l'organisation d'un plébiscite en 1920.

Ces traceurs de frontière sont des diplomates, des militaires mais aussi des géographes, appelés à titre d'experts à se prononcer sur les frontières possibles de la nouvelle Europe. Deux regards de géographes experts se sont portés sur le bassin de Klagenfurt : celui d'Emmanuel de Martonne, expert français au comité d'études chargé d'étudier les questions frontalières par le gouvernement français ${ }^{1}$ et celui de Jovan Cvijić, membre de la délégation officielle des Serbes, Croates et Slovènes. On s'interrogera sur le discours géographique dans le contexte particulier des règlements territoriaux de 1919-1920 suivant trois angles d'analyse : au sein des instances pluridisciplinaires d'expertise tout d'abord, on étudiera la spécificité du discours géographique par rapport à celui de l'historien. Puis on mènera la comparaison entre deux interprétations géographiques du bassin de Klagenfurt conduites par deux experts appartenant à des nations différentes. Enfin, on analysera la confrontation des discours des géographes à la réalité diplomatique lors de la Conférence de la Paix. Pour cela, on s'appuiera sur les sources mentionnées dans la bibliographie de fin d'article.

\section{Confins montagnards versus carrefour alpin}

Dans les travaux du comité d'études français, l'historien Émile Haumant ${ }^{2}$ est chargé de l'expertise des frontières nord de la future Yougoslavie. Il y convoque le principe des nationalités, base primordiale des pourparlers à la Conférence de la Paix, et l'argument des droits historiques. L'historien reconnait tout de suite la complexité d'une telle entreprise dans la région des Alpes orientales : "cette frontière sera difficile à tracer. Elle ne peut correspondre à des limites politiques, ni s'appuyer sur des lignes d'eau ou de sommets ; rarement elle pourra suivre une démarcation tranchée entre deux zones linguistiques ; partout il faudra transiger entre les conditions communément exigées d'une bonne frontière ${ }^{3 "}$. Tracer une bonne frontière signifie pour l'historien s'appuyer sur des lignes : celles de repères topographiques qui renvoient aux linéaments du relief ou bien celles des limites politiques ou linguistiques. Son argumentation se fonde sur l'étude de l'avancée allemande en Carinthie pour

\footnotetext{
${ }^{1}$ Pour des détails sur le comité d'études, le processus de détermination d'une frontière depuis l'expertise géographique jusqu'aux résolutions de la Conférence de la Paix, nous renvoyons à notre maîtrise BOULINEAU E. (1996) reprise en partie dans notre article BOULINEAU E., (2001).

${ }^{2}$ Émile Haumant (1859-1942), était titulaire de la chaire de littérature russe à la faculté de Paris, après des études à l'École des Chartes et aux Langues orientales. Agrégé d'histoire et docteur ès lettres, il était spécialiste de la Russie et des pays slaves. Cf. CHARLE C. (1986), Les professeurs de la faculté des lettres de Paris, Dictionnaire biographique (1909-1939), Paris, INRP-CNRS, p. 103.

${ }^{3}$ HAUMANT É., 1919, p. 533.
} 
déterminer des droits territoriaux historiques. Mais il se heurte à l'indécision de la distribution territoriale entre les deux groupes linguistiques à cause des fluctuations de la colonisation. Les structures politiques ne lui permettent pas davantage de trancher : au Moyen-âge les provinces de Carniole, de Carinthie et de Styrie sont des marches militaires à l'extension spatiale fluctuante et à l'époque moderne, les villes sont allemandes mais les campagnes slovènes.

L'historien, suivant le principe des nationalités énoncé, propose alors de s'appuyer sur la limite linguistique : "on ne pourra tracer la frontière de demain qu'en suivant la limite linguistique d'aujourd'hui". Certes, il y aurait bien quelques entorses avec des poches germaniques en territoire slovène mais ces enclaves essentiellement urbaines pourraient très vite s'accommoder de leur nouveau sort: "ces forteresses du germanisme changeront de caractère aussi vite que l'a fait Lioubliana (Laybach) au XIXe siècle, sous la seule pression du sentiment slave réveillét". Les statistiques ethniques autrichiennes montrent en effet que de nombreux urbains recensés comme Allemands conservent le droit de domicile dans des communes à majorité slovène. C'est le cas pour Maribor (Marbourg) : parmi les 24601 habitants recensés, 13239 d'entre eux ont des attaches dans des villages slovènes alors que seulement 4062 Slovènes ont été comptés. L'historien conclut à la validité de la ligne ethnographique actuelle pour guider la frontière.

Cet enchevêtrement de lignes et de fronts, tel que l'a décrit É. Haumant, révèle un bassin de Klagenfurt conçu comme un espace où se sont confrontées constructions politiques, influences linguistiques et vagues migratoires. Là où l'historien cherche à repérer des lignes stables, et de ce fait se heurte ici à une grande complexité, le géographe cherche à prendre en compte l'espace, l'organisation territoriale de ce qu'il y a entre les lignes. Lucien Gallois oppose à É. Haumant la nécessité de prendre en compte les relations économiques et les moyens de communication car, selon lui, l'argument ethnographique n'est pas l'unique critère. Autrement dit la répartition des flux, la circulation des produits contribuent à créer une cohérence territoriale. La géographie s'intéresse à la construction du territoire, à l'œuvre de cohésion interne plutôt qu'à la seule enveloppe externe. C'est là une façon de congédier le débat entre frontière naturelle et frontière artificielle comme l'avait fait Camille Vallaux (1911) pour s'intéresser aux structures territoriales. Le bassin de Klagenfurt apparait dès lors comme un carrefour alpin où se croisent des voies de communication et des flux marchands, alors que l'historien y voit une zone montagnarde de confins où se sont heurtées diverses influences. Ce souci du géographe pour les configurations spatiales transparaît dans les deux études du bassin de Klagenfurt menées par deux experts au titre de délégations nationales différentes : E. de Martonne pour la France et J. Cvijić pour la partie serbe.

\section{Regards géographiques croisés sur un carrefour alpin}

E. de Martonne (1873-1955) et J. Cvijic ${ }^{5}$ (1865-1927) sont deux géomorphologues réputés qui s'investissent dans le règlement des questions frontalières au nom d'un investissement patriotique certain. La participation d'E. de Martonne au comité d'études dès sa formation, son engagement scientifique comme secrétaire des travaux d'expertise traduisent ses sentiments patriotiques. J. Cvijić de son côté a milité très tôt pour la formation d'un État des Slaves du sud contre la politique pangermaniste d'expansion territoriale des empires centraux (Korinman, 1991). Il est d'ailleurs contraint de fuir son pays en 1915 devant l'avancée des troupes austro-hongroises. Sa reconnaissance

\footnotetext{
${ }^{4}$ Ibid. p. 534.

${ }^{5}$ J. Cvijić est né à Loznica, dans un des foyers de la renaissance culturelle serbe. Spécialiste de géomorphologie, il passe 4 ans à l'Université de Vienne et soutient une thèse en 1893 sur les phénomènes karstiques. À son retour en Serbie, il fonde l'institut de géographie à l'Université de Belgrade en 1894 puis la Société serbe de géographie en 1910. Ses recherches en géomorphologie le conduisent à parcourir la péninsule balkanique mais ses travaux s'orientent peu à peu vers la géographie humaine avec, à partir de 1902, le début d'une longue série d'études sur les populations balkaniques pour l'Académie des sciences. Recteur de la nouvelle Université de Belgrade en 1905 puis de nouveau en 1919, J. Cvijic a contribué au développement d'une école de géographie serbe. Président de l'Académie des sciences serbe en 1921, il y engage des chercheurs de diverses disciplines dans l'étude de la péninsule balkanique (Vasović, 1980).
} 
internationale par les milieux scientifiques s'accroît grâce à ses publications en anglais, allemand et français, notamment celles qui concernent les populations slaves de la Macédoine (Wilkinson, 1951).

Autre point commun, les deux géographes sont des hommes de terrain, rompus à la pratique des excursions et des missions de recherche. Le géographe français prenait de nombreuses notes sur des carnets qu'il complétait de croquis et de photos (Joseph et Robic, 1987) et a institué en France la pratique de l'excursion scientifique (Delfosse, 2001). Suite à une mission, E. de Martonne signe un article sur les processus d'érosion glaciaire dans les Tauern d'Autriche et les Carpates (de Martonne, 1911). Il réutilise ses qualités d'observation pour rédiger les volumes de la Géographie Universelle consacrés à l'Europe centrale. Il y décrit avec précision l'habitat du bassin de Klagenfurt : "Dans la Carinthie surtout, les hameaux et les fermes isolées, avec la petite maison au toit pentu et les dépendances en désordre tout autour, font penser aux Balkans" (de Martonne, 1931, p.471). J. Cvijić, nourrit aussi ses études de la pratique du terrain : il a ainsi parcouru la péninsule balkanique pour ses travaux sur le karst (Bosnie, Herzégovine, Monténégro), les formations lacustres (Macédoine, Albanie actuelle, Grèce) ou l'étude des glaciations (Bulgarie, Grèce) mais aussi pour ses recherches de géographie humaine. Plus encore, le géographe serbe a formé des équipes de recherche, constituées d'étudiants mais aussi de prêtres ou d'instituteurs, pour étudier les populations des Balkans. Ces travaux constitueront une source volumineuse pour la rédaction de son ouvrage le plus connu, la Péninsule balkanique publié en français en 1918 (Vasović, 1980).

Les deux hommes se connaissaient, à titre universitaire comme spécialistes de géographie physique tout d'abord, mais aussi à titre personnel. J. Cvijić est de 8 ans l'aîné d'E. de Martonne mais ils ont tous deux suivi les enseignements d'Albrecht Penck à Vienne : le Serbe de 1889 à 1893 pour son doctorat, le Français lors d'une bourse d'études de 1896-1897 qui le mène dans les grandes universités allemandes (Delfosse, 2001). En 1917 et 1918, J. Cvijić qui a fui alors la Serbie occupée, est invité à professer en Sorbonne aux côtés de son collègue E. de Martonne. Les deux géographes enfin se côtoient dans les séances du comité d'études : le Français écoute les requêtes du représentant serbe. La stature de J. Cvijić a d'ailleurs frappé l'esprit du jeune Georges Chabot, alors secrétaire de séance, qui évoque "un homme coiffé d'un chapeau à larges bords faisant figure de montagnard farouche" (Chabot, 1972). Les affinités pro-serbes, enfin, rapprochent les deux hommes : J. Cvijić croit au rôle fédérateur de la Serbie dans l'union des Salves du sud ; E. de Martonne, à l'instar d'universitaires français tel Ernest Denis, est gagné "d'un véritable engouement pour la Serbie" (Bariéty, 2000). Géographes éminents mais aux appartenances nationales différentes, quelle pensée de la frontière austro-slovène développent-ils ? Mobilisent-ils les mêmes critères géographiques à cette époque d'affirmation universitaire de la discipline ?

\section{Le bassin de Klagenfurt, Alpes de la Mitteleuropa ou balkan slovène?}

Dans de rapides descriptions les géographes ne s'embarrassent pas de considérations morphologiques mais évoquent le modelé glaciaire pour expliquer le paysage lacustre (Cvijić, 1919) ou la fertilité des sols (de Martonne, 1919a). En revanche, ils insistent sur le site de la ville de Klagenfurt, au centre d'un bassin entouré de massifs alpins. En effet, leurs expertises géographiques montrent que les enjeux de la frontière austro-slovène se cristallisent sur cette situation de carrefour du bassin.

Cette dépression intra-montagnarde est ainsi le lieu de confrontation entre les influences linguistiques et culturelles slave et germanique. C'est là toute sa complexité : "le bassin de Klagenfurt est un carrefour qui sera vivement disputé ; il l'a toujours été entre les races slave et germanique (...). Actuellement le bassin de Klagenfurt est coupé en diagonale par la limite des langues." (de Martonne, $1919 b$, p.544). Les géographes s'emploient alors à dénombrer, localiser et comprendre les enjeux ethnographiques. Ils dénoncent tous deux la politique de germanisation issue du mouvement pangermaniste qui s'est renforcée dans la deuxième moitié du XIXe siècle et dont les statistiques ethniques et leurs évolutions font état. E. de Martonne reproduit les données du recensement autrichien de 1910, réputé fiable, mais doute de l'importance des germanophones. Reprenant à son compte l'analyse d'E. Haumant évoquée ci-dessus, le géographe français critique le principe de la langue d'usage retenu dans le recensement autrichien au détriment de la langue maternelle. Les individus bilingues pour des raisons économiques conversent en allemand et sont dès lors comptabilisés comme Autrichiens, gonflant ainsi les effectifs des germanophones. Le géographe 
prend le parti de compter ensemble les catégories slave et "autres" ; il dénombre ainsi pour la Carinthie une population slovène qui atteint un cinquième des 390000 habitants, mais dont la distribution spatiale est très inégale selon les arrondissements.

J. Cvijić partage le même avis avec davantage de virulence car les mouvements de population sont au centre même de sa pensée géographique de l'espace balkanique. Le géographe serbe écrit l'histoire des nombreuses invasions et vagues migratoires qui ont parcouru la péninsule balkanique. Ce qui fait la particularité de son approche, c'est l'accent mis sur un type de mouvements migratoires qu'il qualifie "d'immigrations serbo-croates" (Cvijić, 1919) et qui recouvrent ce qu'il a appelé auparavant "mouvements métanastasiques" ${ }^{6 "}$ (Cvijić, 1918). "Ces petites migrations incessantes de la péninsule balkanique vers le Basin pannonnien" (Cvijić, 1919, p. 12), sous la poussée turque ou sous des contraintes économiques, ont ponctué les siècles et contribué aux contacts entre les peuplades slaves. J. Cvijić fait ainsi la part belle à la masse serbe, ballottée de lieu en lieu au gré des flux et des reflux des empires ottoman et austro-hongrois, dans la formation d'une conscience nationale des Slaves du sud : "les immigrants balkaniques communiquèrent à l'ancienne population leurs mœurs, leur folklore ; ils lui apprirent les chants nationaux d'origine serbe et lui apportèrent la conscience nationale très développée qui s'était élaborée au cours des siècles dans les pays balkaniques" (ibid., p.15). Mais il déplore que l'œuvre entamée par les Serbes soit fragilisée par la germanisation et la magyarisation à partir de la deuxième moitié du XIXe siècle. Les Slovènes des Alpes orientales subissent alors une accélération de la colonisation allemande : "les montagnes et les rivières dirigées de l'Ouest en Est s'opposaient à la poussée germanique vers le Sud ; mais la puissante nation allemande a pu parvenir à surmonter ces obstacles" (ibid. p.17). La germanisation pénètre la population slovène mais pour le géographe serbe "il s'agit de sentiments passagers et récemment acquis" (ibid. p.22). J. Cvijić se livre ainsi à une démonstration nationaliste de l'unité des Slaves du Sud devant l'oppression extérieure. C'est aussi l'objet du second livre de La Péninsule balkanique intitulé "caractères psychiques des Yougoslaves". Sous forme de présentation anthropologique du caractère des populations balkaniques, le livre constitue un manifeste de la solidarité entre Yougoslaves. Le géographe y présente en effet une diversité de "types" et de "variétés" psychiques mais qui sont toutes la déclinaison d'une seule et même ethnie, celle des Slaves du sud, au premier rang de laquelle figure le type dinarique représentatif des Serbes.

A l'appui de son expertise, chacun dresse une carte ethnographique en couleurs de la région afin de convaincre par l'impression visuelle. Les deux géographes sont d'ailleurs d'éminents cartographes reconnus pour leur savoir-faire (Palsky, 2001 et Wilkinson, 1951). La carte d'E. de Martonne au 1500 $000^{\text {e }}$ s'étend de la Carinthie au Banat (de Martonne, 1919c). La carte d'état-major du service géographique de l'armée française constitue le fond topographique sur lequel sont surimposées les limites des arrondissements autrichiens. Bien plus précis, J. Cvijić a dressé un carton cartographique à l'échelle du $200000^{\text {e }}$ centré sur le bassin de Klagenfurt (Cvijić, 1919). Les procédés statistiques de traitement cartographique en pourcentage sont utilisés dans les deux cas, mais l'interprétation de la carte qui en résulte change du tout au tout. Le Français souhaite représenter les arrondissements contestés (carte 2), là où l'attribution de la majorité numérique aux Autrichiens ou aux Slovènes est impossible (à peu près $50 \%$ de la population) ou litigieuse (environ 30\%). Ceux-ci correspondent en effet à une bande médiane le long de la Drave, tantôt en deçà tantôt au-delà de la rivière. Au contraire, la carte de J. Cvijić tend à démontrer la progressive confiscation territoriale slovène par les Autrichiens (carte 3). Il trace le front de germanisation en figurant la limite ethnographique du peuplement allemand en 1851 et montre la progressive mise en minorité numérique des Slaves en représentant des îlots germanisés (moins de $50 \%$ de la population est slovène) enchâssés dans des zones à majorité slovène. Autrement dit, là où le géographe français représente une zone ethnographique complexe où l'indécision de la distribution ethnique apparaît, le géographe serbe dénonce la colonisation germanique qui a créé des "poches de germanisation". Là où, de fait, E. de Martonne congédie le principe des nationalités impossible à appliquer ici pour fixer la frontière, J. Cvijić milite pour la réhabilitation des droits territoriaux slovènes au nom de ce même principe. Il en

\footnotetext{
${ }^{6}$ Le terme est forgé sur une racine grecque qui signifie "mouvement d'habitat". Il faut entendre par là des migrations de voisinage (George, 1991).
} 
résulte un désaccord entre les deux géographes sur l'importance à accorder aux arguments économiques et stratégiques.

Or le bassin de Klagenfurt est un carrefour ferroviaire où se croisent des routes qui mènent de Vienne et Graz en pays autrichien à Ljubljana en Carniole, Trieste et Fiume sur le littoral adriatique ; du col du Tarvis communiquant avec l'Italie à Maribor en empruntant d'est en ouest la vallée de la Drave (carte XY). J. Cvijić plaide résolument pour une frontière ethnographique et accorde peu d'importance aux faits de circulation. À ses yeux, les chemins de fer autrichiens ont contribué à l'oppression économique germanique : "L'administration, les tribunaux, l'école, l'église, l'armée contribuèrent à propager la langue allemande. A cette action s'ajoutaient celles des Vereine, des associations allemandes de propagande, de tourisme même attiré vers ces régions pittoresques, de la vie économique et de la civilisation allemandes" (Cvijić, 1919, p. 22). E. de Martonne creuse davantage l'étude du trafic économique et des intérêts stratégiques. Certes, les Autrichiens détiennent le pouvoir économique (commerce, industrie, banque) mais l'analyse précise des flux de marchandises en gare de Klagenfurt révèle une prédominance du trafic avec les pays slovènes. Cette solidarité économique entre le bassin alpin et les pays slaves de la plaine danubienne plaide pour son attribution au futur État yougoslave : "il est possible que la séparation de Vienne (...), amène d'abord une crise, mais il peut en résulter un développement de la vie industrielle plus autonome (...). Entre les mains d'un (1)tat yougoslave, les hauts-fourneaux auraient plus de chance de rester allumés, l'importation du charbon s'organisant par Fiume" (de Martonne, 1919a, p.545). On retrouve ici une géographie qui étudie les configurations territoriales internes et non les limites externes, qui analyse les flux et les cohésions spatiales créées. C'est la conception vidalienne de la région, formulée par P. Vidal de La Blache dans La France de l'Est en 1917, qui ressort ici.

Deux lectures donc de la frontière à tracer dans ces Alpes orientales, qui témoignent de deux approches différentes. J. Cvijić dissimule un discours nationaliste sous une argumentation géographique remarquable d'érudition. Pour lui, le bassin de Klagenfurt appartient de plein droit à la péninsule balkanique : il en a connu les mouvements de populations, les oppressions étrangères, les tentatives d'intégration aux empires. Il le définit comme un balkan (montagne en turc) slovène. E. de Martonne adopte le style plus didactique et plus détaché de l'expert : "les conditions géographiques et économiques seront appelées à jouer un grand rôle. Nous allons essayer d'en donner un aperçu" (de Martonne, 1919a, p. 544). Ses études de géographie physique sur les Alpes orientales les rattachent au système des Carpates (1911), et donc à l'Europe centrale. Mais au point de vue économique, le géographe refuse d'intégrer le bassin de Klagenfurt à cet espace placé sous la coupe des puissances germaniques. On peut y voir aussi le refus d'une lecture géopolitique, celle de la Mitteleuropa, caractéristique de l'école française de géographie du début du siècle.

\section{Frontière de géographes versus frontière de diplomates}

À La Conférence de la Paix qui s'ouvre à Paris en janvier 1919, la frontière austro-slovène donne lieu à d'âpres débats, consignés en partie dans le recueil des actes de la conférence? ${ }^{7}$ La question est étudiée par la commission territoriale chargée des affaires roumaines et yougoslaves, composée de délégués de chaque pays allié (Royaume Uni, France, États-Unis, Italie). On y retrouve nos deux géographes, qui n'ont ici qu'une voix consultative. Les tentatives de trouver un accord frontalier se heurtent à la difficulté de tracer la ligne ethnique et aux désaccords entre les délégations alliées ${ }^{8}$. La Grande-Bretagne et les États-Unis sont très attachés au principe des nationalités et refusent de se satisfaire d'un projet français fondé sur des considérations économiques et stratégiques. L'Italie quant à elle exige de garder une voie de chemin de fer directe avec l'Autriche et la Hongrie, sans transiter par le territoire slovène car elle possède un sérieux contentieux territorial à propos de l'Istrie et de la Carniole avec les Yougoslaves. L'éventualité d'un plébiscite est rapidement retenue mais il reste à s'accorder sur la définition du territoire de consultation. E. de Martonne défend une position pro-serbe : il insiste en effet pour que le bassin de Klagenfurt soit attribué provisoirement à la Yougo-slavie car

\footnotetext{
${ }^{7}$ Archives du Ministère des affaires Etrangères, Conférence de la Paix, recueil des actes, volume 53.

${ }^{8}$ ibid. PV 15 du 11 mars 1919.
} 
il craint que l'Autriche n'y exerce une coercition sur les populations dans l'attente de la consultation'. Une nouvelle audition de la délégation serbe, croate et slovène le 20 mai 1919 donne la parole à $J$. Cvijić : il réitère la position serbe de délimiter la frontière dans le bassin de Klagenfurt sur le critère de la langue. E. de Martonne, présent à la séance, ajoute foi aux données chiffrées de son collègue et loue son "honnêteté scientifique"10. Mais les plénipotentiaires de la commission demeurent divisés : la France et la Grande-Bretagne estiment que la population est en grande partie slovène et que le bassin de Klagenfurt doit être attribué au royaume serbe, croate et slovène plutôt qu'au vaincu autrichien. Les Américains dans le doute prônent le plébiscite, l'Italie enfin refuse la solution du plébiscite, de peur de devoir en essuyer un dans la région très contestée de l'Istrie, et réclame une révision totale des tracés pour préserver ses intérêts ferroviaires stratégiques. Devant l'impossibilité de trouver un accord entre les délégations, la commission territoriale se défait du dossier des frontières du Bassin de Klagenfurt pour le confier à l'instance supérieure du Conseil suprême des Alliés ${ }^{11}$ qui décide le plébiscite. La consultation populaire est inscrite dans le traité de Saint-Germain du 10 septembre 1919 qui ratifie les frontières de l'Autriche.

Le plébiscite est organisé le 10 octobre 1920. La zone de consultation avait été préalablement divisée en deux : la première zone au sud de la Drave, dans l'axe du lac de Wörth fut consultée en premier et donna un résultat favorable à l'Autriche : 22025 voix contre 15025 à la Yougoslavie (de Martonne, 1921). Le commentaire des résultats par de Martonne montre une pointe d'amertume : "Ce résultat couronne l'œuvre de germanisation systématiquement entreprise depuis cinquante ans dans la Carinthie méridionale, conduite par des sociétés puissantes que la propagande slovène avait commencé seulement à combattre dans les dernières années" (ibid. p.80). Le géographe avait déjà exprimé auparavant sa réticence à la solution du plébiscite dans cette région en la qualifiant "d'expédient" (de Martonne, 1920). On ne peut s'empêcher ici de faire la comparaison avec le cas de L'Alsace-Lorraine, soumise à la domination allemande depuis 1870, pour laquelle E. de Martonne s'était opposé à toute éventualité de plébiscite (Ter Minassian, 1997). Pour sa part, J Cvijić, membre de la commission internationale de contrôle du plébiscite, démissionne pour marquer son désaccord avec cette solution (Korinman, 1991). Les deux géographes, dont les expertises divergeaient, se retrouvent dans l'amertume du résultat final. Leurs interventions, qui plaidaient pour des raisons différentes au rattachement du bassin à la Slovénie, ont été vaines devant les décisions diplomatiques. Et pourtant, c'est là une des rares frontières de 1920 qui ne sera pas remise en cause en 1945, en dépit de l'occupation nazie de la Slovénie durant la seconde Guerre mondiale et du retour de la politique pangermaniste. Dans ces Alpes orientales qui forment un entre-deux entre une péninsule balkanique aux accents slaves et une Mitteleuropa à consonance germanique, les habitants du bassin de Klagenfurt ont choisi l'Europe centrale et son potentiel économique au retard balkanique ponctué de crises. L'indépendance de la Slovénie en 1991 consacre aussi son retour à l'Europe centrale après des décennies d'appartenance à la Yougoslavie balkanique. Les Alpes, s'il fallait encore s'en convaincre après les démonstrations d'E. de Martonne et de J. Cvijić, n'ont rien d'une barrière et encore moins d'une frontière naturelle ${ }^{12}$.

\section{Bibliographie :}

\section{Documents sources :}

CVIJIĆ J., 1918.- La péninsule balkanique, Paris, Colin, 529p., 3 cartes h.t.

CVIJIĆ J., 1919.- Frontière septentrionale des Yougoslaves, Paris, 1919, 30p., 3 cartes h.t.

\footnotetext{
${ }^{9}$ Ibid. PV 21 du 9 mai 1919.

${ }^{10}$ Ibid. PV 25 et 26 du 20 mai 1919.

${ }^{11}$ Pour des précisions sur le processus de décision à la Conférence de la Paix, voir BOULINEAU E. (2001).

${ }^{12}$ On peut aussi s'en convaincre en montrant que la province de Carinthie a élu un gouverneur d'extrême droite en la personne de J. Haider, porte-parole de thèses contre les réfugiés de l'Est.
} 
HAUMANT E., 1919.- La frontière septentrionale de l'État yougo-slave. Travaux du comité d'études, Questions européennes, Paris, Imprimerie nationale, p.533-543.

MARTONNE E. de, 1911.- Étude morphologique des Alpes orientales (Tauern) et des Karpates septentrionales (Tatra). Bulletin de géographie historique et descriptive, n³, p.387-406.

MARTONNE E. de, 1919a.- Note sur les conditions géographiques de la limite des langues slave et germanique dans les Alpes. Travaux du comité d'études, Questions européennes, Paris, Imprimerie nationale, p.544-546.

MARTONNE E. de, 1919b.- Note sur la carte des nationalités dans les régions frontières de la Yougoslavie. Travaux du comité d'études, Questions européennes, Paris, Imprimerie nationale, p.547-552.

MARTONNE E. de (dir.), 1919c.- Travaux du comité d'études. Atlas. Questions européennes, Paris, Service géographique de l'armée, 21 planches.

MARTONNE E. de, 1920.- Le traité de Saint-Germain et le démembrement de l'Autriche. Annales de Géographie, t.XXIX, n¹57, p.1-11.

MARTONNE E. de, GALLOIS L., 1921.- Résultats des plébiscites et des décisions arbitrales. Annales de Géographie, t.XXX, n¹63, p.73-80.

MARTONNE E. de, 1931.- Géographie universelle : Europe centrale, Tome 4, volume 2, Paris, Colin, 845p.

VALLAUX C.,1911.- Le sol et l'@tat, Paris, Doin, 420p.

Ouvrages et articles :

ANCEL J., 1930.- Peuples et nations des Balkans. Paris, Colin, 220p.

BARIÉTY J., 2000.-La France et la naissance du Royaume des serbes, Croates et Slovènes, 19141919. Relations internationales, $\mathrm{n}^{\circ} 103$, p. 307-327.

BOULINEAU E., 1996.- La conception des frontières par les géographes français et sa mise en application lors des traités de paix de 1919-1920 sur l'Europe centrale et orientale. Maitrise de géographie sous la direction de V. Rey, Université Paris 1, 130p. dactylographiées.

BOULINEAU E., 2001.- Un géographe traceur de frontières : Emmanuel de Martonne et la Roumanie. L'Espace géographique, $\mathrm{n}^{\circ} 3$ (à paraître).

CHABOT G., 1972.- La géographie appliquée à la Conférence de la Paix en 1919 : une séance francopolonaise. Mélanges offerts au Professeur Meynier : la pensée géographique contemporaine, SaintBrieuc, PUB, p. 101-105.

DELFOSSE C., 2001.- Emmanuel de Martonne, tisseur de réseaux internationaux de géographes. BAUDELLE G., OZOUF-MARIGNIER M-V., ROBIC M-C., Géographes en pratiques (1870-1945) : le terrain, le livre, la Cité, Rennes, Presses universitaires de Rennes, p.189-205.

GEORGE P., 1991.- Le destin de la Yougoslavie : déterminisme géographique ou malédiction historique ? La réponse de Jovan Cvijić (mai 1918). Hérodote, nº3, p.47-51.

JOSEPH M., ROBIC M-C., 1987.- Autour des papiers d'E. de Martonne. Acta geographica, ${ }^{\circ} 72$, p.37-65. 
KORINMAN M., 1991.- L'Autriche, l'Allemagne et les Slaves du Sud. Hérodote, n63, p. 52-65.

PALSKY G., 2001.- L'esprit, l'œil, la main. Emmanuel de Martonne et la cartographie. BAUDELLE G., OZOUF-MARIGNIER M-V., ROBIC M-C., Géographes en pratiques (1870-1945) : le terrain, le livre, la Cité, Rennes, Presses universitaires de Rennes, p.269-276.

TER MINASSIAN T., 1997.- Les géographes français et la délimitation des frontières balkaniques à la Conférence de la Paix en 1919. Revue d'histoire moderne et contemporaine, n 44, p.252-286.

VASOVIĆ M., 1980.- Jovan Cvijić (1865-1927). Geographers bibliographical studies, vol. 4, p. 2532.

WILKINSON H.R., 1951.- Maps and politics : a review of the ethnographic cartography of Macedonia, Liverpool, University press, 366p. 


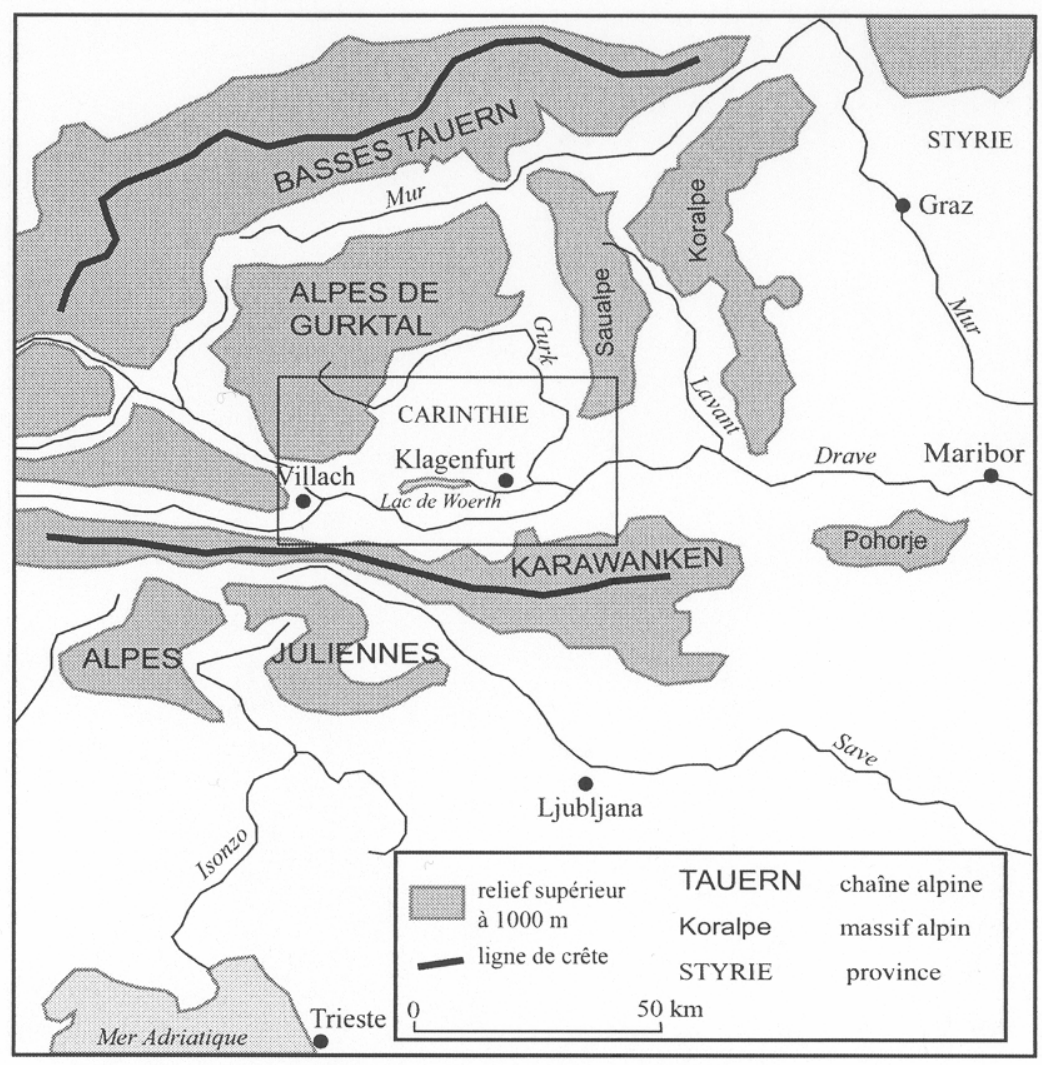

Carte 1 : Situation du bassin de Klagenfurt Situation of the region of Klagenfurt 


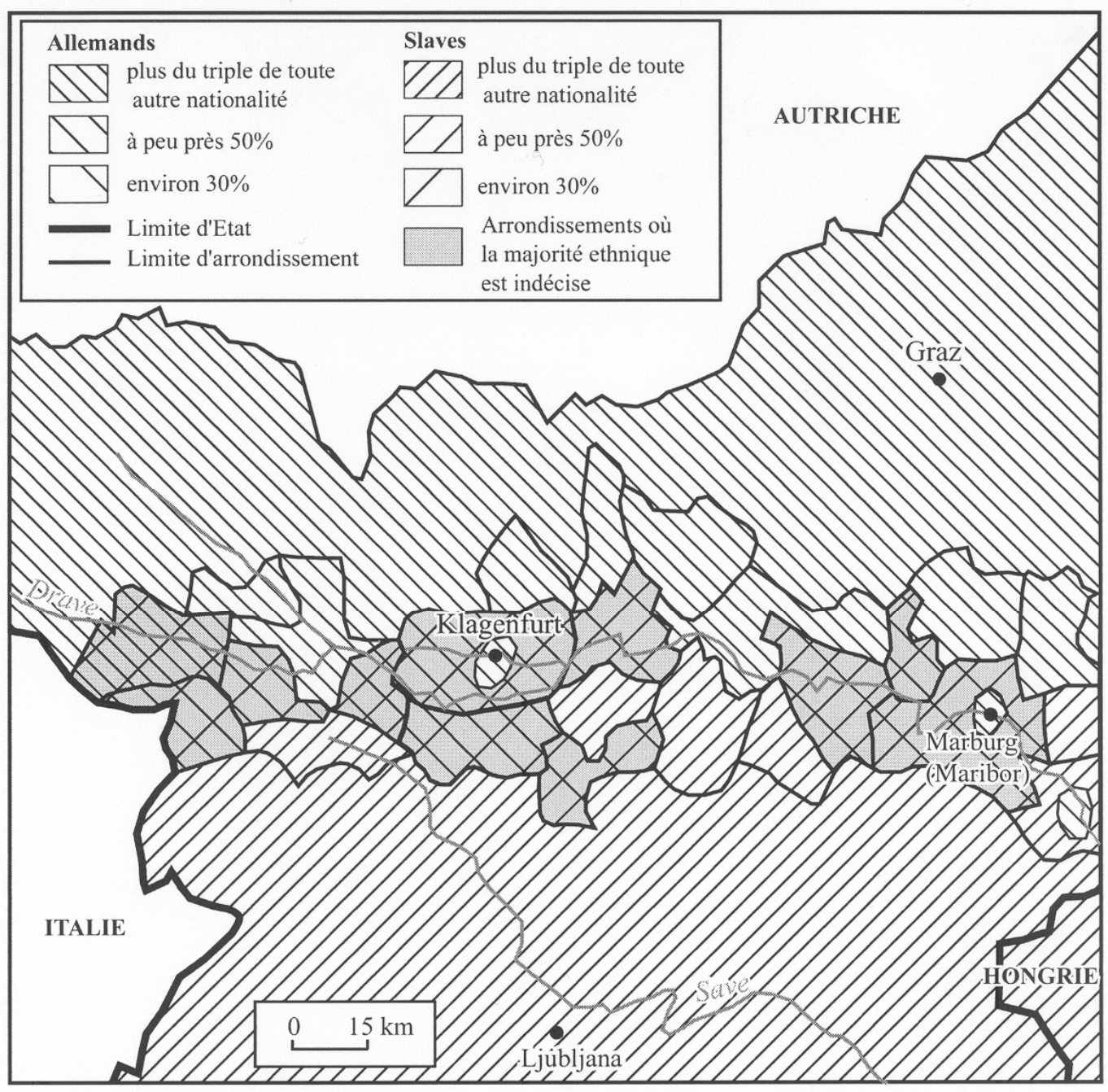

Carte 2 : Répartition des nationalités d'après E.de Martonne (1919c) Nationality patterns according to E. de Martonne (1919c) 


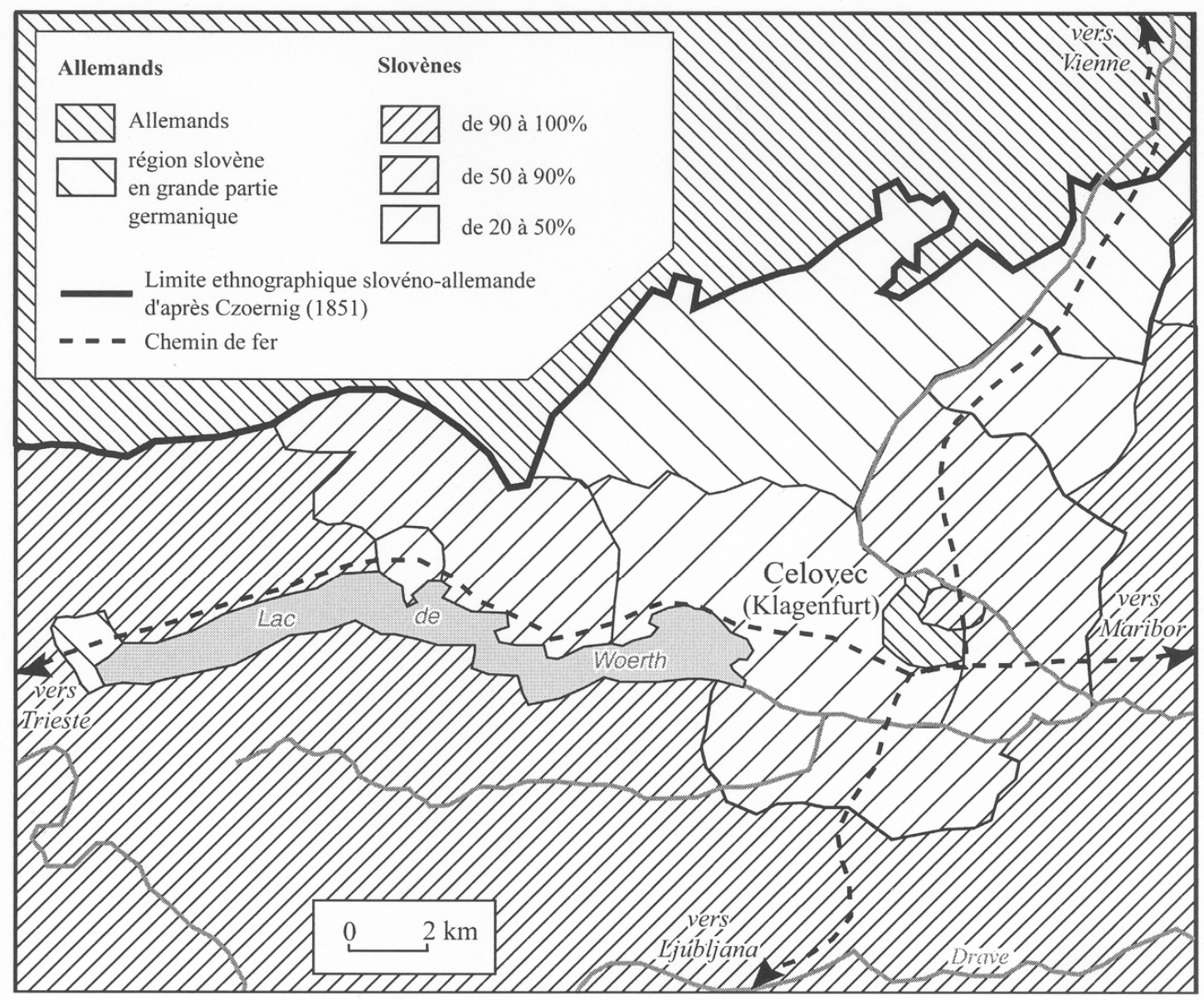

Carte 2 : Les environs de Celovec (Klagenfurt) d'après J. Cvijic (1919)

The region of Celovec (Klagenfurt) according to J. Cvijic (1919)

Nationality patterns according to E. de Martonne (1919c) 